\title{
Influence of a low frequency vibration on a long-wave Marangoni instability in a binary mixture with the Soret effect
}

\author{
I. S. Fayzrakhmanova, ${ }^{1}$ S. Shklyaev, ${ }^{2,3}$ and A. A. Nepomnyashchy ${ }^{1,4}$ \\ ${ }^{1}$ Department of Mathematics, Technion-Israel Institute of Technology, Haifa 32000, Israel \\ ${ }^{2}$ Department of Chemical Engineering, California Institute of Technology, Pasadena, California 91125, USA \\ ${ }^{3}$ Institute of Continuous Media Mechanics, Ural Branch of the Russian Academy of Sciences, \\ Perm 614013, Russia \\ ${ }^{4}$ Minerva Center for Nonlinear Physics of Complex Systems, Technion-Israel Institute of Technology, \\ Haifa 32000, Israel
}

(Received 17 May 2010; accepted 25 August 2010; published online 19 October 2010)

\begin{abstract}
We study the influence of a low frequency vibration on a long-wave Marangoni convection in a layer of a binary mixture with the Soret effect. A linear stability analysis is performed numerically by means of the Floquet theory; several limiting cases are treated analytically. Competition of subharmonic, synchronous, and quasiperiodic modes is considered. The vibration is found to destabilize the layer, decreasing the stability threshold. Also, a vibration-induced mode is detected, which takes place even for zero Marangoni number. (C) 2010 American Institute of Physics.

[doi:10.1063/1.3489411]
\end{abstract}

\section{INTRODUCTION}

Vibration is a widespread method of control used in engineering processes on different scales from acoustic mixing in microfluidic devices ${ }^{1}$ to the ultrasound method in oil prospecting and extraction. ${ }^{2}$ A variety of vibration-induced phenomena could be roughly divided into two important classes: (i) the averaged influence and (ii) the parametric excitation. In the case (i), the vibration period is small compared to a characteristic time of fluid motion; the most striking examples are the acoustic streaming, ${ }^{3}$ thermal vibrational convection, ${ }^{4}$ and steady surface deformation emerging at a free surface/interface in layered systems. ${ }^{5-7}$ In the case (ii), the period of the external forcing is comparable with a characteristic time of the fluid system. The Faraday instability ${ }^{8}$ (see reviews in Refs. 5 and 9) and the parametric excitation in a thermal convection ${ }^{4}$ can be mentioned as examples of such phenomena. Since the present paper focuses on the parametric instability in a convective system, we discuss the latter subject in more details.

The buoyancy convection in a pure liquid under a modulated gravity field was analyzed in Refs. 10-14. In particular, the linear stability problem for a layer under a gravity modulation was studied. ${ }^{13,14}$ Of a special interest is the vibration influence on the layer of a binary mixture, ${ }^{15-18}$ where an oscillatory convection takes place in the absence of vibration and, hence, resonant phenomena occur under the periodic forcing.

In comparison with the buoyancy convection, the surface-tension-driven convection in oscillatory external fields is less studied. We refer to Refs. 19-25 on this subject. A keen interest here is in the resonant excitation of the oscillatory eigenmodes stemming from the surface deformation (see reviews on the oscillatory Marangoni instabilities in Refs. 26-28). Usually, a surface deflection considerably complicates the analysis, especially for the nonlinear problem. However, there also exists an example of oscillatory
Marangoni convection in a system with a nondeformable surface, it is the thermo- and solutocapillary convection in a layer of binary mixture. To the best of our knowledge, no vibration influence has been studied for such a system.

In a contradistinction to a monotonic Marangoni instability, which can be often investigated analytically, the onset of an oscillatory convection usually can be studied only numerically. Therefore, an analytical study of the influence of a small amplitude vibration on the oscillatory mode, similar to that known for the Mathieu equation, ${ }^{29}$ cannot be carried out in the case of convection. The long-wave convection is a rare exception, for which even an oscillatory mode can be treated analytically. For instance, both monotonic and oscillatory modes were studied for the long-wave Marangoni convection in a layer of a binary liquid in the presence of the Soret effect. ${ }^{30}$ The present paper is devoted to the analysis of the influence of vibrations on the stability of the above mentioned system.

The paper is organized as follows. We start with the problem formulation in Sec. II. Section III provides a derivation of an amplitude equation to be solved and briefly describes a method that we use to treat that linear problem. Limiting cases are analyzed in Sec. IV. The numerical results are discussed in Sec. V; Sec. VI contains the summary.

\section{PROBLEM FORMULATION AND GOVERNING EQUATIONS}

Consider an infinite layer of an incompressible binary liquid of a thickness $d_{*}$, which is infinite in the horizontal directions $x_{*}$ and $y_{*}$. (Hereafter the subscript $*$ denotes dimensional variables.)

The lower rigid boundary $\left(z_{*}=0\right)$ is subject to oscillations in $z_{*}$-direction with an amplitude $b_{*}$ and a frequency $\Omega_{*}$. A transverse temperature gradient $-a_{*}$ is maintained through the layer. The temperature gradient induces a gradi- 
ent of the solute concentration due to the presence of the Soret effect. The mass flux is given by

$$
\mathbf{J}_{\mathbf{m}}=-\rho_{*} D_{*}\left(\nabla C_{*}+\alpha_{*} \nabla T_{*}\right),
$$

here $\rho_{*}, D_{*}, \alpha_{*}, T_{*}$, and $C_{*}$, are the density of the fluid, mass diffusivity, Soret coefficient, temperature, and solute concentration, respectively.

The upper free surface is nondeformable. Both the surface tension $\sigma_{*}$ and density $\rho_{*}$ linearly depend on the temperature $T_{*}$ and solute concentration $C_{*}$

$$
\begin{aligned}
& \sigma_{*}=\sigma^{(0)}-\sigma_{T}\left(T_{*}-T_{*}^{(0)}\right)+\sigma_{C}\left(C_{*}-C_{*}^{(0)}\right), \\
& \rho_{*}=\rho^{(0)}\left[1-\beta_{T}\left(T_{*}-T_{*}^{(0)}\right)-\beta_{C}\left(C_{*}-C_{*}^{(0)}\right)\right],
\end{aligned}
$$

wherein $C_{*}^{(0)}$ and $T_{*}^{(0)}$ are the reference values of $T_{*}$ and $C_{*}$, respectively, $\sigma_{T}=-\partial \sigma / \partial T_{*}, \sigma_{C}=\partial \sigma / \partial C_{*}, \beta_{C}$, and $\beta_{T}$ are the solutal and the thermal expansion coefficients, respectively. In the framework of the Boussinesq approximation, the last two terms in Eq. (3) are rather small. The vibration-induced acceleration is assumed to be large with respect to the gravity acceleration, which allows us to take into account the vibrational convection neglecting the gravity-induced one. Quantitative relations which determine the limits for the abovementioned approximations are discussed in Appendix A.

We choose $\kappa_{*} / d_{*}, d_{*}^{2} / \kappa_{*}, d_{*}, a_{*} d_{*}, \sigma_{T} a_{*} d_{*} / \sigma_{C}$, and $\rho^{(0)} \nu_{*} \kappa_{*} / d_{*}^{2}$ as the scales for the velocity, time, length, temperature, solute concentration, and pressure, respectively. Here $\kappa_{*}$ is the thermal diffusivity and $\nu_{*}$ is the kinematic viscosity.

The dimensionless boundary value problem governing the motion of the binary liquid is

$$
\nabla \cdot \mathbf{v}=0
$$

$$
\frac{1}{\mathrm{P}}\left(\frac{\partial \mathbf{v}}{\partial t}+\mathbf{v} \cdot \nabla \mathbf{v}\right)=-\nabla p+\nabla^{2} \mathbf{v}+\mathbf{e}_{\mathbf{z}} b(\varphi C+T) \cos \omega t,
$$

$$
\begin{aligned}
& \frac{\partial T}{\partial t}+\mathbf{v} \cdot \nabla T=\nabla^{2} T, \\
& \frac{1}{\mathrm{~L}}\left(\frac{\partial C}{\partial t}+\mathbf{v} \cdot \nabla C\right)=\nabla^{2} C+\chi \nabla^{2} T, \\
& \mathbf{v}=0, \quad \partial_{z} T=-1, \quad \partial_{z} C=\chi \text { at } z=0, \\
& w=0, \quad \partial_{z} \mathbf{u}=-\mathrm{M}_{2}(T-C), \\
& \partial_{z} T=-1, \quad \partial_{z} C=\chi \text { at } z=1,
\end{aligned}
$$

where $\mathbf{v}=\mathbf{u}+w \mathbf{e}_{z}, \mathbf{e}_{z}=(0,0,1), \nabla_{2}$ is a two-dimensional projection of the gradient operator onto the $x-y$ plane, $p$ is an addition to a linear pressure distribution $\rho^{(0)}\left(z_{*}\right.$ -1) $b_{*} \Omega_{*}^{2} \cos \Omega_{*} t_{*}$, and the rest of notations are conventional.

The boundary value problem, Eqs. (4)-(9), is governed by seven dimensionless parameters

$$
\begin{gathered}
\mathrm{P}=\frac{\nu_{*}}{\kappa_{*}}, \mathrm{~L}=\frac{D_{*}}{\kappa_{*}}, \mathrm{M}=\frac{\sigma_{T} a_{*} d_{*}^{2}}{\nu_{*} \rho^{(0)} \kappa_{*}}, \quad \chi=\frac{\alpha_{*} \sigma_{C}}{\sigma_{T}}, \\
\varphi=\frac{\beta_{C} \sigma_{T}}{\beta_{T} \sigma_{C}}, \quad b=\frac{b_{*} \Omega_{*}^{2} d_{*}^{4} \beta_{T} a_{*}}{\kappa_{*} \nu_{*}}, \omega=\frac{\Omega_{*} d_{*}^{2}}{\kappa_{*}},
\end{gathered}
$$

which are the Prandtl, Lewis, Marangoni, and Soret numbers, dimensionless parameter containing ratios of the thermal and solutal expansions and thermal and solutal surface tension coefficients, amplitude and frequency of vibration, respectively. Hereafter we deal with the low frequency approximation, setting $\omega \ll 1$. Note, that the dimensional vibration amplitude $b_{*}$ should be larger than $d_{*}$ to ensure finite values of $b$ in this case (see Appendix A). It should be also noted that for the most of the liquid binary mixtures the Lewis number is rather small. However, we do not assume $\mathrm{L}$ being asymptotically small.

This boundary value problem has an obvious solution corresponding to the quiescent fluid

$$
\begin{aligned}
& \mathbf{v}_{0}=0, T_{0}=-z+T_{00}, C_{0}=\chi z+C_{00}, \\
& p_{0}=\frac{b}{2} \cos \omega t\left(\varphi_{\chi} z^{2}+p_{01} z\right)+p_{00},
\end{aligned}
$$

where constants $T_{00}, C_{00}, p_{01}$, and $p_{00}$ are unimportant for the further analysis, $\varphi_{\chi} \equiv \varphi \chi-1$. Below we consider the linear stability of this base state.

\section{AMPLITUDE EQUATIONS AND METHOD OF SOLUTION}

We study the stability of the base state given by Eq. (11) with respect to infinitesimal disturbances. Owing to the obvious symmetry reasons, a two-dimensional stability problem is considered. This allows us to introduce the streamfunction $\psi$ according to the relations $w=-\partial_{x} \psi, u=\partial_{z} \psi$, where $u$ is the $x$-component of the fluid velocity.

Linearizing Eqs. (4)-(9) around the base state and representing an arbitrary field of normal perturbations $f$ in the form

$$
f(t, x, z)=e^{i k x} \widetilde{f}(t, z),
$$

with a wavenumber $k$, we arrive at the following set of equations:

$$
\begin{aligned}
& \frac{1}{\mathrm{P}} \frac{\partial \Delta \tilde{\psi}}{\partial t}=\Delta^{2} \tilde{\psi}-i k(\tilde{T}+\varphi \widetilde{C}) b \cos \omega t, \\
& \frac{\partial \tilde{T}}{\partial t}=\Delta \tilde{T}-i k \tilde{\psi}, \\
& \frac{1}{\mathrm{~L}} \frac{\partial \widetilde{C}}{\partial t}=\Delta \tilde{C}+\chi \Delta \widetilde{T}+\frac{1}{\mathrm{~L}} i k \chi \tilde{\psi}, \\
& \tilde{\psi}=\partial_{z} \tilde{\psi}=\partial_{z} \tilde{T}=\partial_{z} \tilde{C}=0 \text { at } z=0,
\end{aligned}
$$




$$
\begin{aligned}
& \widetilde{\psi}=\partial_{z} \tilde{T}=\partial_{z} \widetilde{C}=0, \\
& \partial_{z}^{2} \widetilde{\psi}=-i k \mathrm{M}(\widetilde{T}-\widetilde{C}) \text { at } z=1,
\end{aligned}
$$

where $\Delta \equiv \partial_{z}^{2}-k^{2}$.

In the absence of the vibration $b=0$, this stability problem amounts to the limiting case of the zero Biot number $B=0$ of the problem analyzed in Ref. 30 . In the cited paper, both monotonic and oscillatory modes of a long-wave instability were found, and the present paper is devoted to the vibration impact on these modes. It has been shown that for disturbances with small wavenumbers,

$$
k=\varepsilon K, 0<\varepsilon \ll 1,
$$

the frequency of oscillations generated by the instability is $O\left(\varepsilon^{2}\right)$. As mentioned in the introduction, the parametric excitation takes place when the external forcing is of the order of the internal frequencies of the system. Therefore, the appropriate scaling for finding a parametric excitation of oscillations is $\omega=O\left(\varepsilon^{2}\right)$. It is convenient to define the small parameter of expansions as $\varepsilon=\sqrt{\omega}$, hence $\omega=\varepsilon^{2}$. Similarly to Ref. 30, we introduce

$$
\tau=\varepsilon^{2} t, \tilde{\psi}=\varepsilon^{2} \tilde{\Psi},
$$

and expand the fields of perturbations in power series in small $\varepsilon$

$$
(\tilde{\psi}, \tilde{T}, \widetilde{C})=\left(\widetilde{\Psi}_{0}, \widetilde{T}_{0}, \widetilde{C}_{0}\right)+\left(\widetilde{\Psi}_{2}, \widetilde{T}_{2}, \widetilde{C}_{2}\right) \varepsilon^{2}+\cdots .
$$

Substituting the expansions into Eqs. (13)-(17), one obtains in the zeroth order

$$
\begin{aligned}
& \frac{\partial^{4} \widetilde{\Psi}_{0}}{\partial z^{4}}=i K\left(\widetilde{T}_{0}+\varphi \widetilde{C}_{0}\right) b \cos \tau=0, \\
& \frac{\partial^{2} \widetilde{T}_{0}}{\partial z^{2}}=0, \frac{\partial^{2} \widetilde{C}_{0}}{\partial z^{2}}=0, \\
& \widetilde{\Psi}_{0}=\partial_{z} \widetilde{\Psi}_{0}=\partial_{z} \widetilde{T}_{0}=\partial_{z} \widetilde{C}_{0}=0 \text { at } z=0, \\
& \widetilde{\Psi}_{0}=\partial_{z} \widetilde{T}_{0}=\partial_{z} \widetilde{C}_{0}=0, \\
& \partial_{z}^{2} \widetilde{\Psi}_{0}=-i K \mathrm{M}\left(\widetilde{T}_{0}-\widetilde{C}_{0}\right) \text { at } z=1 .
\end{aligned}
$$

Solution in the zeroth order is given by

$$
\begin{aligned}
\widetilde{T}_{0}= & a_{1}(\tau), \widetilde{C}_{0}=a_{2}(\tau), \\
\widetilde{\Psi}_{0}= & \frac{i K \mathrm{M}}{4}\left(\widetilde{T}_{0}-\widetilde{C}_{0}\right) z^{2}(1-z) \\
& +\frac{i K b}{24} \cos \tau\left(\widetilde{T}_{0}+\varphi \widetilde{C}_{0}\right)\left(z^{4}-\frac{5}{2} z^{3}+\frac{3}{2} z^{2}\right),
\end{aligned}
$$

where $a_{1}(\tau)$ and $a_{2}(\tau)$ are yet unknown. The boundary value problem in the second order is presented in Appendix B. The solvability condition for this problem (see also Appendix B) results in the set of ordinary differential equations for $a_{1}(\tau)$ and $a_{2}(\tau)$

$$
\begin{aligned}
\dot{a}_{1}= & a_{1} K^{2}(m-1+A \cos \tau)-a_{2} K^{2}(m-\varphi A \cos \tau), \\
\dot{a}_{2}= & -a_{1} K^{2}(m \chi+\chi \mathrm{L}+\chi A \cos \tau) \\
& +a_{2} K^{2}(\chi m-\mathrm{L}-\varphi \chi A \cos \tau),
\end{aligned}
$$

where the derivatives with respect to $\tau$ are denoted by the dots, $A=b / 320$ and $m=\mathrm{M} / 48$.

It is convenient to rewrite this problem in terms of perturbations of the density $\rho$ and surface tension $-h$

$$
\rho=a_{1}+\varphi a_{2}, h=a_{1}-a_{2} .
$$

Eliminating $h$ from the further analysis, we arrive at the following equation:

$$
\ddot{\rho}-\alpha \dot{\rho}+\varphi_{\chi} K^{2} A(\rho \cos \tau)_{\tau}-\rho(\Theta+\Phi A \cos \tau)=0,
$$

where subscript $\tau$ denotes the time derivative, $\alpha=K^{2} \alpha_{k}$ $=K^{2}[m(\chi+1)-\mathrm{L}-1], \quad \Theta=K^{4} \Theta_{k}=K^{4}\left(m \chi_{L}-\mathrm{L}\right), \quad \Phi=K^{4} \Phi_{k}$ $=-K^{4}\left[\varphi_{\chi}(\mathrm{L}+1)+1\right]$, and $\chi_{L} \equiv \chi+\mathrm{L}+\chi \mathrm{L}$.

If $\Theta<0$, this second order ordinary differential equation for $\rho$ is similar to the well-known Mathieu equation with a damping. Later on, we solve Eq. (27) analytically in certain limiting cases and numerically, applying the Floquet theory.

According to the Floquet theory, we represent the solution of Eq. (27) in the form

$$
\rho(\tau)=e^{\Lambda \tau} R_{0}(\tau),
$$

where $R_{0}(\tau)$ is $2 \pi$-periodic function, $\Lambda=\Lambda_{r}+i \Lambda_{i}$ is the complex Floquet exponent. Following the Floquet theory (see, for instance, Ref. 29), one can find the Floquet exponents $\Lambda_{n}$ $(n=1,2)$ or the Floquet multipliers, $\mu_{n}=e^{2 \pi \Lambda_{n}}$, numerically. (We assume $\Lambda_{1 r}>\Lambda_{2 r}$.) At the stability boundary, the real part of $\Lambda_{1}$ vanishes or, equivalently, $\left|\mu_{1}\right|=1$. It is usual to classify the perturbations according to the value of $\Lambda_{1 i}$ at the stability boundary in the following way: (i) subharmonic mode with $\mu_{1}=-1$ or $\Lambda_{1 i}=1 / 2$; (ii) synchronous mode with $\mu_{1}=1, \Lambda_{1 i}=0$; (iii) quasiperiodic modes with a pair of complex-conjugate multipliers $\left|\mu_{1,2}\right|=1,0<\left|\Lambda_{1 i}\right|<1 / 2$.

\section{LIMITING CASES}

In the present section, we consider some analytically tractable limiting cases.

\section{A. Small amplitude analysis}

Setting $A=0$ in Eq. (27), one can readily reproduce the results of Ref. 30, where both the oscillatory and monotonic instabilities were found in the absence of the vibration. Seeking solution in the form

$$
\rho=\frac{1}{2} C_{1} e^{i \gamma \tau}+\text { c.c. },
$$

where c.c. means complex conjugated terms, we arrive at

$$
\gamma^{2}+i \gamma \alpha_{k} K^{2}+\Theta_{k} K^{4}=0 \text {. }
$$

The oscillatory instability $(\gamma \neq 0$ at the stability border $)$ occurs within the interval

$$
-1<\chi<\chi_{o}, \quad \chi_{o}=-\frac{\mathrm{L}^{2}}{1+\mathrm{L}+\mathrm{L}^{2}} .
$$



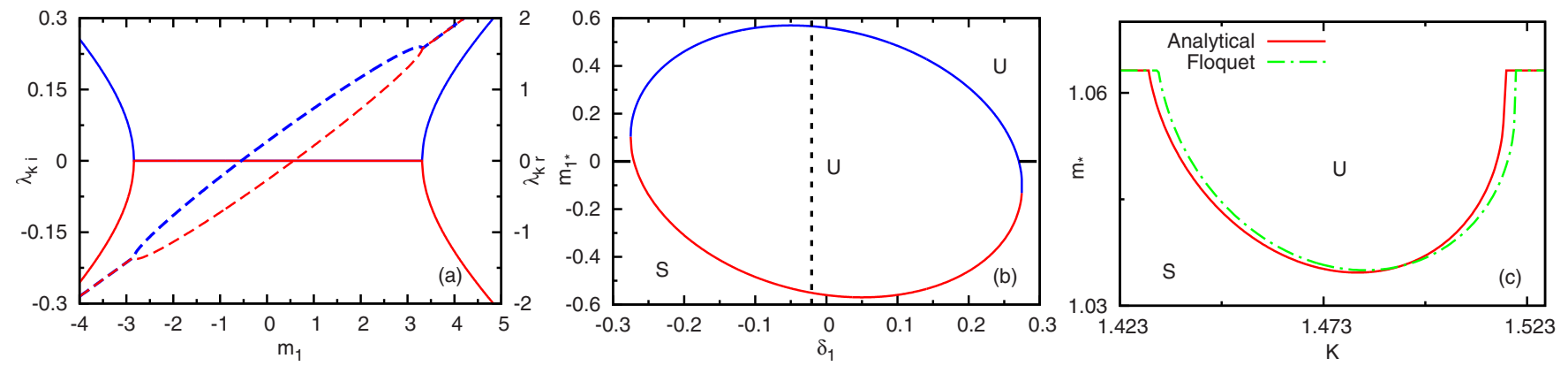

FIG. 1. (Color online) Results on the linear stability for $\gamma(K) \approx 1 / 2 ; \chi=-0.05\left(K_{*}=1.47\right)$. Panel (a) $\lambda_{k r}\left(m_{1}\right)$ and $\lambda_{k i}\left(m_{1}\right)$ at $\delta_{1}=-0.0207$ [vertical dashed line in panel (b)]. Solid and dashed lines correspond to the imaginary and real part of $\lambda_{k}$, respectively. Panel (b): $m_{1 *}\left(\delta_{1}\right)$ at $\lambda_{k r}=0$. Panel (c): subharmonic instability zone (above the curves) for $A=0.05$. The capitals "S" and "U" correspond to stability and instability areas.

The stability boundary is determined by the relation

$$
m_{o}=\frac{1+\mathrm{L}}{1+\chi}
$$

The dispersion relation for neutral perturbations is

$$
\gamma(K)=K^{2} \Omega_{k}, \quad \Omega_{k} \equiv \sqrt{-\frac{\chi\left(1+\mathrm{L}+\mathrm{L}^{2}\right)+\mathrm{L}^{2}}{1+\chi}} .
$$

The difference between the obtained expression for $\Omega_{k}$ and Eq. (26) in Ref. 30 is caused by a different choice of time scales.

The critical Marangoni number for the monotonic instability ( $\gamma=0$ at the stability boundary) is

$$
m_{m}=\frac{\mathrm{L}}{\chi_{L}} .
$$

This mode is critical either at $\chi>\chi_{o}$ or $\chi<\chi_{2}$, where

$$
\chi_{2}=-\frac{\mathrm{L}}{1+\mathrm{L}} \text {. }
$$

In the latter case $m_{m}<0$.

At small values of $A$, the critical Marangoni number should be close to the values given by either Eq. (32) or Eq. (34) and the perturbation theory can be applied to determine the small corrections to the threshold value. For this goal we expand $\rho$ and $m$ in powers of $A$ and introduce slow time scales

$$
\begin{aligned}
& \rho=\rho_{0}+A \rho_{1}+\ldots, \\
& m=m_{0}+A m_{1}+\ldots, \tau_{n}=A^{n} \tau,
\end{aligned}
$$

where $m_{0}$ is given by either Eq. (32) or Eq. (34).

The solution in the zeroth order is given by Eq. (29), where subscript " 0 " should be prescribed for both $\rho_{0}$ and $\tau_{0}$; $C_{1}$ depends now on slow time variables $\tau_{1}, \tau_{2}$, etc.

Besides, keeping in mind the definitions of $\Theta_{k}$ and $\alpha_{k}$, we obtain for the oscillatory mode

$$
\Theta_{k}=-\Omega_{k}^{2}+\chi_{L}\left(m-m_{0}\right), \quad \alpha_{k}=(\chi+1)\left(m-m_{0}\right),
$$

and for the monotonic mode

$$
\Theta_{k}=\chi_{L}\left(m-m_{0}\right), \quad \alpha_{k}=\alpha_{0}+(\chi+1)\left(m-m_{0}\right),
$$

where $\alpha_{0}=-\chi\left(1+\mathrm{L}+\mathrm{L}^{2}\right) \chi_{L}^{-1}$.
First we analyze the impact of the vibration on the oscillatory mode. In the limit $A \rightarrow 0$ the subharmonic mode takes place when $\gamma\left(K_{*}\right)=1 / 2$, i.e., the wavenumber $K_{*}$ $=1 / \sqrt{2 \Omega_{k}}$ is selected by this resonant condition. In the vicinity of $K_{*}$ we can write

$$
\gamma(K)=K^{2} \Omega_{k} \approx \frac{1}{2}+\delta_{1} A K_{*}^{2},
$$

i.e., $\delta_{1} A=2 \Omega_{k}\left(K / K_{*}-1\right)$.

In the first order, we have

$$
\begin{aligned}
\hat{L} \rho_{1}= & \frac{\partial \rho_{0}}{\partial \tau_{0}} K_{*}^{2} m_{1}(\chi+1)-\frac{\partial\left(\rho_{0} \cos \tau\right)}{\partial \tau_{0}} K_{*}^{2} \varphi_{\chi}-2 \frac{\partial^{2} \rho_{0}}{\partial \tau_{0} \partial \tau_{1}} \\
& +\rho_{0} K_{*}^{4}\left(m_{1} \chi_{L}+\Phi_{k} \cos \tau\right),
\end{aligned}
$$

where we have introduced the operator $\hat{L}=\partial_{\tau_{0}}^{2}+\gamma^{2}$.

Elimination of secular terms gives

$$
\begin{aligned}
2 i \gamma \frac{\partial C_{1}}{\partial \tau_{1}}= & \frac{C_{1}^{*}}{2} K_{*}^{2}\left[i \varphi_{\chi}(\gamma-1)+K_{*}^{2} \Phi_{k}\right] e^{-2 i \delta_{1} K_{*}^{2} \tau_{1}} \\
& +m_{1} K_{*}^{2}\left[i \gamma(\chi+1)+K_{*}^{2} \chi_{L}\right] C_{1} .
\end{aligned}
$$

Hereafter the asterisk denotes the complex conjugation.

A standard replacement $\widetilde{C}_{1}=C_{1} \exp \left(i \delta_{1} K_{*}^{2} \tau_{1}\right)$ transforms Eq. (41) into a linear differential equation with constant coefficients. Replacing $d / d \tau_{1}$ by $\lambda=\lambda_{k} K_{*}^{2}$, we arrive at the quadratic equation for $\lambda_{k}=\lambda_{k r}+i \lambda_{k i}$

$$
\begin{aligned}
\Omega_{k}^{2} & {\left[2 \lambda_{k}-(\chi+1) m_{1}\right]^{2}-\frac{\Omega_{k}^{2} \varphi_{X}^{2}}{4}+\left(\chi_{L} m_{1}-2 \Omega_{k} \delta_{1}\right)^{2}-\frac{\Phi_{k}^{2}}{4} } \\
& =0 .
\end{aligned}
$$

Typical variation of $\lambda_{k}$ with $m_{1}$ is presented in Fig. 1(a). Within a certain interval $m_{l}<m_{1}<m_{u}$ [see the horizontal solid line in the Fig. 1(a)] both growth rates are real. Beyond this interval a complex conjugate pair of $\lambda_{k}$ solves Eq. (42). First consider the subharmonic mode with $\lambda_{k i}=0$ (recall that $\Lambda_{i}=1 / 2+A \lambda_{k i} K_{*}^{2}$ ). Setting $\lambda_{k}=0$ in Eq. (42), we obtain a quadratic equation, which couples $m_{1 *}$ and $\delta_{1}$. (Hereafter the asterisk for $m$ denotes the marginal stability threshold.) This stability boundary is an ellipse on the plane $m_{1 *}-\delta_{1}$ with its principal axes tilted to $m_{1 *}=0$ and $\delta_{1}=0$, see Fig. 1(a). At the lower line $m_{1 *}=m_{l}\left(\delta_{1}\right)$ the smaller growth rate vanishes, whereas at the upper line $m_{1 *}=m_{u}\left(\delta_{1}\right)$ the larger one becomes zero. For $\delta_{1}<0$ there is a short interval where $m_{l}$ 
$>0$, and there the quasiperiodic mode is critical. On the contrary, when $\delta_{1}>0$ there is an interval of $\delta_{1}$, where $m_{l}$ $<m_{u}<0$ and thus the stability boundaries for both the subharmonic modes are below that for the quasiperiodic mode. For larger $\left|\delta_{1}\right|$ no real values of $m_{1 *}$ are possible, and the subharmonic mode disappears. In this case $\lambda_{k i} \neq 0$ at the stability boundary, i.e., the quasiperiodic mode becomes critical. For this mode

$$
m_{1 *}=0, \quad \lambda_{k i}^{2}=\delta_{1}^{2}-\frac{\Phi_{k}^{2}+\varphi_{\chi}^{2} \Omega_{k}^{2}}{16 \Omega_{k}^{2}} .
$$

Therefore, far enough from the resonance we have $m_{*}-m_{o}$ $=O\left(A^{2}\right)$ and only the frequency of the neutral perturbations changes. [Moreover, numerical calculations show that the stability threshold is given by Eq. (32) in any order with respect to $A$ for the quasiperiodic mode.]

Thus, the subharmonic mode is critical; minimization of $m_{1 *}$ with respect to $\delta_{1}$ provides

$$
m_{1 c}^{\mathrm{sub}}= \pm \frac{\sqrt{\Phi_{k}+\varphi_{\chi}^{2} \Omega_{k}^{2}}}{2 \Omega_{k}(\chi+1)}, \quad \delta_{c}^{\mathrm{sub}}=m_{1 c}^{\mathrm{sub}} \frac{\chi_{L}}{2 \Omega_{k}} .
$$

The lower sign in the expression for $m_{1 c}^{\text {sub }}$ corresponds to the minimum of the neutral curve. The oscillatory instability exists in the interval of $\chi$ determined by Eq. (31), therefore, $\chi+1>0$ and $-1<\chi_{L}<\mathrm{L} /\left(1+\mathrm{L}+\mathrm{L}^{2}\right)$, since the Lewis number is typically small. Thus, the first correction $m_{1 c}^{\text {sub }}$ is negative and the vibration leads to a destabilization of the layer. As $A$ increases, the critical wavenumber grows at $-1<\chi$ $<\chi_{2}$ and diminishes at $\chi_{2}<\chi<\chi_{o}$.

A typical form of the neutral stability curve $m_{o}+A m_{1 *}$ * for the subharmonic mode is depicted in Fig. 1(c). Horizontal lines in this figure correspond to $m_{1 *}=0$ according to Eq. (43).

For the synchronous mode we have

$$
\begin{aligned}
& \gamma=1+\delta_{2} A^{2} K_{*}^{2}, \quad m=m_{o}+A^{2} m_{2}, \\
& \partial_{\tau}=\partial_{\tau_{0}}+A^{2} \partial_{\tau_{2}}+\ldots,
\end{aligned}
$$

i.e., the wavenumber is close to $K_{*}=1 / \sqrt{\Omega_{k}}$.

In the first order we have

$$
\hat{L} \rho_{1}=-K_{*}^{2} \varphi_{\chi} \frac{\partial}{\partial \tau_{0}}\left(\rho_{0} \cos \tau\right)+\rho_{0} \Phi \cos \tau
$$

The particular solution of the equation contributing the secular terms is

$$
\rho_{1}=\frac{C_{1}}{12}\left[3 \Phi e^{i \delta_{2} \tau_{2} K_{*}^{2}}-\left(\Phi-2 i K_{*}^{2} \varphi_{\chi}\right) e^{2 i \gamma \tau_{0}-i \delta_{2} \tau_{2} K_{*}^{2}}\right]+\text { c.c. },
$$

where $C_{1}$ is the constant entering zero-order solution, Eq. (29). Hereafter we omit solutions of the homogeneous equations, which do not contribute into the secular terms.

In the second order we have

$$
\begin{aligned}
\hat{L} \rho_{2}= & \rho_{1} \Phi \cos \tau-2 \frac{\partial^{2} \rho_{0}}{\partial \tau_{0} \partial \tau_{2}}+\rho_{0} m_{2} K_{*}^{4} \chi_{L} \\
& +K_{*}^{2} \frac{\partial}{\partial \tau_{0}}\left[\rho_{0} m_{2}(\chi+1)-\rho_{1} \varphi_{\chi} \cos \tau\right] .
\end{aligned}
$$

The solvability condition is

$$
\begin{aligned}
\frac{i \Omega_{k}}{K_{*}^{2}} \frac{\partial C_{1}}{\partial \tau_{2}}= & \frac{m_{2} C_{1}}{2}\left[\chi_{L}+i \Omega_{k}(\chi+1)\right]+\frac{C_{1}\left(\Phi_{k}^{2}+\varphi_{\chi}^{2} \Omega_{k}^{2}\right)}{12 \Omega_{k}^{2}} \\
& +\frac{\left(\Phi_{k}-i \Omega_{k} \varphi_{\chi}\right) \Phi_{k} C_{1}^{*} e^{-2 i \delta_{2} K_{*}^{2} \tau_{2}}}{8 \Omega_{k}^{2}} .
\end{aligned}
$$

It provides a quadratic equation for $m_{2}\left(\delta_{2}\right)$ that yields the stability boundary

$$
F_{1}^{2}+\frac{\Phi_{k}^{2}}{4} F_{1}+F_{2}^{2}+\frac{\varphi_{\chi}^{2} \Phi_{k}^{2} \Omega_{k}^{2}}{64}=0,
$$

where

$$
\begin{aligned}
& F_{1}=-\Omega_{k}^{3} \delta_{2}-\frac{\Phi_{k}^{2}}{24}+\frac{\varphi_{\chi}^{2} \Omega_{k}^{2}}{12}+\frac{m_{2} \Omega_{k}^{2} \chi_{L}}{2}, \\
& F_{2}=\Omega_{k}^{3} \lambda_{k}-\frac{m_{2}(\chi+1) \Omega_{k}^{3}}{2},
\end{aligned}
$$

here $\lambda=\lambda_{k} K_{*}^{2}$ is the growth rate in the slow time $\tau_{2}$.

One can see that this equation is quadratic in both $\lambda$ and $m_{2}$, its structure is very similar to the previous case, cf. Eq. (42); the dependences of $\lambda\left(m_{2}\right), m_{2 *}\left(\delta_{2}\right)$, and $m_{*}(K)=m_{o}$ $+A^{2} m_{2 *}(K)$ have the forms similar to those shown in Fig. 1.

We now proceed to the vibration impact on the monotonic mode. In this case $\gamma=0$ in Eq. (29) and consequently $\rho_{0}=C_{1}\left(\tau_{1}, \tau_{2}, \ldots\right), m_{0}$ is determined by Eq. (34), i.e., Eq. (38) for $\Theta_{k}$ and $\alpha_{k}$ is valid. Substituting the expansion given by Eq. (36) into Eq. (27) in the first order, we obtain

$$
\begin{aligned}
\frac{\partial^{2} \rho_{1}}{\partial \tau_{0}^{2}}-\alpha_{0} K^{2} \frac{\partial \rho_{1}}{\partial \tau_{0}}= & \rho_{0}\left(\varphi_{\chi} K^{2} \sin \tau+\Phi \cos \tau\right)+\alpha_{0} K^{2} \frac{\partial \rho_{0}}{\partial \tau_{1}} \\
& +\chi_{L} m_{1} K^{4} \rho_{0} .
\end{aligned}
$$

Elimination of the secular terms at the stability boundary $\left(m_{1}=m_{1 *}, \quad \partial_{\tau_{1}}=0\right)$ gives

$$
m_{1 *}=0 \text {, }
$$

and the particular solution in the first order is

$$
\begin{aligned}
\rho_{1}= & -C_{1} \frac{\Phi\left(K^{2} \alpha_{0} \sin \tau_{0}+\cos \tau_{0}\right)}{\left(K^{4} \alpha_{0}^{2}+1\right)} \\
& -C_{1} \frac{K^{2} \varphi_{\chi}\left(\sin \tau_{0}-K^{2} \alpha_{0} \cos \tau_{0}\right)}{\left(K^{4} \alpha_{0}^{2}+1\right)} .
\end{aligned}
$$

In the second order we arrive at 


$$
\begin{aligned}
\frac{d^{2} \rho_{2}}{d \tau_{0}^{2}}-\alpha_{0} K^{2} \frac{d \rho_{2}}{d \tau_{0}}= & \rho_{1} \Phi \cos \tau-\varphi_{\chi} K^{2} \frac{d\left(\rho_{1} \cos \tau\right)}{d \tau_{0}} \\
& +\alpha_{0} K^{2} \frac{d \rho_{0}}{d \tau_{2}}+\chi_{L} m_{2} K^{4} \rho_{0} .
\end{aligned}
$$

Elimination of secular terms at the stability boundary $\left(m_{2}\right.$ $\left.=m_{2^{*}}, \quad \partial_{\tau_{2}}=0\right)$ gives

$$
m_{2 *}=\frac{K^{4} \Phi_{k}\left(\Phi_{k}-\varphi_{\chi} \alpha_{0}\right)}{2 \chi_{L}\left(K^{4} \alpha_{0}^{2}+1\right)},
$$

therefore, $m_{2 *}$ varies monotonically from zero at $K=0$ to $\Phi_{k}\left(\Phi_{k}-\varphi_{\chi} \alpha_{0}\right) / 2 \chi_{L} \alpha_{0}^{2}$ at large $K$. The ratio $m_{2 \infty}$ of the latter value to $m_{m}=L / \chi_{L}$ after simplification is given by

$$
m_{2 \infty}=\frac{\chi[\varphi \chi(\mathrm{L}+1)-\mathrm{L}][\varphi \mathrm{L}(\chi+1)+1] \chi_{L}}{2 \mathrm{~L}\left[\chi\left(1+\mathrm{L}+\mathrm{L}^{2}\right)+\mathrm{L}^{2}\right]^{2}} .
$$

One can see that the expression has a singularity at $\chi=\chi_{o}$, where it asymptotically tends to the infinity. At four values of $\chi$

$$
\chi_{1}=-\left(1+\frac{1}{\varphi \mathrm{L}}\right), \quad \chi_{3}=0, \quad \chi_{4}=\frac{\mathrm{L}}{\varphi(1+\mathrm{L})},
$$

and $\chi_{2}$ given by Eq. (35), the expression changes its sign.

For instance, for $\varphi=1$ the correction, determined by Eq. (57), is positive for $\chi<\chi_{1}, \chi_{2}<\chi<0$, and $\chi_{4}<\chi$ and negative for $\chi_{1}<\chi<\chi_{2}$ or $0<\chi<\chi_{4}$. In the former case (positive $m_{2 \infty}$ ), the long-wave mode with $K=0$ is critical, whereas in the latter case (negative $m_{2 \infty}$ ) the short-wave mode is critical. These limiting cases, for finite vibration amplitudes, are analyzed in Secs. IV B and IV C, respectively. It is obvious that no resonant excitation of the oscillatory convection is possible in these two limits, thus we consider the vibration impact on the monotonic mode only.

\section{B. Solution for small $K$}

We now proceed to the limit of small $K$ representing $\rho$ and $m$ as power series in $K^{2}$ :

$$
\begin{aligned}
& \rho=\rho_{0}+K^{2} \rho_{1}+K^{4} \rho_{2}+\ldots, \\
& m_{*}=m_{0 *}+K^{2} m_{1 *}+K^{4} m_{2 *}+\cdots .
\end{aligned}
$$

In the zeroth order, one obtains

$$
\ddot{\rho}_{0}=0, \quad \rho_{0} \equiv 1 .
$$

In the first order we have

$$
\ddot{\rho}_{1}=\rho_{0} \varphi_{\chi} A \sin \tau, \rho_{1}=-\varphi_{\chi} A \sin \tau .
$$

In the second order

$$
\begin{aligned}
\ddot{\rho}_{2}= & \dot{\rho}_{1} \alpha_{k}\left(m_{0 *}\right)-\varphi_{X} A\left(\rho_{1} \cos \tau\right)_{\tau} \\
& +\rho_{0}\left[\Theta_{k}\left(m_{0 *}\right)+\Phi_{k} A \cos \tau\right] .
\end{aligned}
$$

The secular (time-independent) terms should vanish in the right-hand side of Eq. (63), hence we obtain

$$
\Theta_{k}\left(m_{0 *}\right)=0, m_{0 *}=\frac{\mathrm{L}}{\chi_{L}}=m_{m},
$$

i.e., the long-wave limit within Eq. (27) gives the stability threshold in the absence of the vibration. This, in particular, means that the vibration can only destabilize the layer. Besides, we are able to use the expressions for $\alpha_{k}$ and $\Theta_{k}$ given by Eq. (38).

Solution for $\rho_{2}$ has a form

$$
\rho_{2}=-\frac{\left(\varphi_{\chi} A\right)^{2} \cos 2 \tau}{4}+A\left(\alpha_{0} \varphi_{\chi}-\Phi_{k}\right) \cos \tau \text {. }
$$

In the third order

$$
\begin{aligned}
\ddot{\rho}_{3}= & \dot{\rho}_{1}(\chi+1) m_{1 *}+\dot{\rho}_{2} \alpha_{0}-\varphi_{\chi} A\left(\rho_{2} \cos \tau\right)_{\tau} \\
& +\rho_{1} \Phi_{k} A \cos \tau+\rho_{0} m_{1 *} \chi_{L} .
\end{aligned}
$$

Eliminating the unique secular term, we obtain $m_{1 *}=0$.

It can be shown that $\rho_{3}$ does not produce secular terms in the further analysis, and, hence, it is not presented here.

In the fourth order, we obtain

$$
\begin{aligned}
\ddot{\rho}_{4}= & \dot{\rho}_{1}(\chi+1) m_{2 *}-\varphi_{\chi} A\left(\rho_{3} \cos \tau\right)_{\tau}+\rho_{2} \Phi_{k} A \cos \tau \\
& +\dot{\rho}_{3} \alpha_{0}+\rho_{0} m_{2 *} \chi_{L} .
\end{aligned}
$$

The solvability condition reads

$$
m_{2 *}=-\frac{\Phi_{k} A^{2}}{2} \frac{\left[m_{m} \varphi_{\chi}(\chi+1)+1\right]}{\chi_{L}} .
$$

It is worth noting that $m_{2 *} K^{4}$ provides exactly the same result as one derived for small $A$, cf. Eq. (56) at $K \ll 1$. Therefore, the conditions of the long-wave instability [minimum of $m_{*}(K)$ at $K=0$ ] for arbitrary $A$ are exactly the same as they have been formulated at the end of Sec. IV A.

For $\varphi=1$, the long-wave minimum takes place at $\chi$ $<\chi_{1}, \chi_{2}<\chi<0$, or $\chi>\chi_{4}$, whereas the maximum of the marginal stability curve at $K=0$ occurs for $\chi_{1}<\chi<\chi_{2}$ or 0 $<\chi<\chi_{4}$.

\section{Large $K$, WKB approximation}

It should be noted that because the analysis is done in the framework of the lubrication approximation, the case $K$ $\gg 1$ corresponds to an intermediate asymptotics rather than a true short-wave asymptotics. A full stability problem for arbitrary values of the unscaled wavenumber $k$ will be a subject of a future separate analysis. The limit $K \gg 1$ provides an essential (but only sufficient) condition of the short-wave instability. Nevertheless, this case should be treated in the framework of Eq. (27), since it provides an estimation for the domain of parameters, where more expensive computations at finite $k$ are needed.

Owing to the dispersion relation, Eq. (30), the growth rate is proportional to $K^{2}$, i.e., at large $K$ characteristic time of evolution is small in comparison with the vibration period $2 \pi$. Thus, the Wentzel-Kramers-Brillouin (WKB) approximation is appropriate.

According to the WKB approximation, one can present $\rho$ in a form 


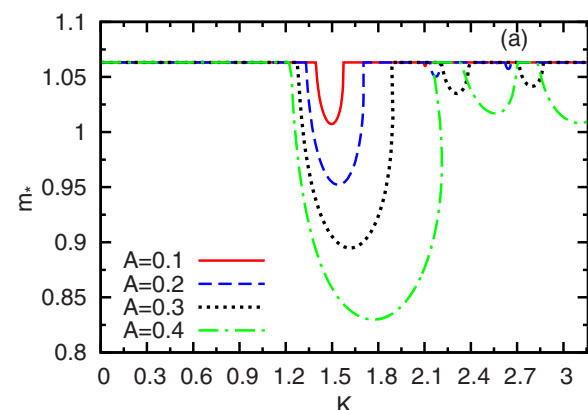

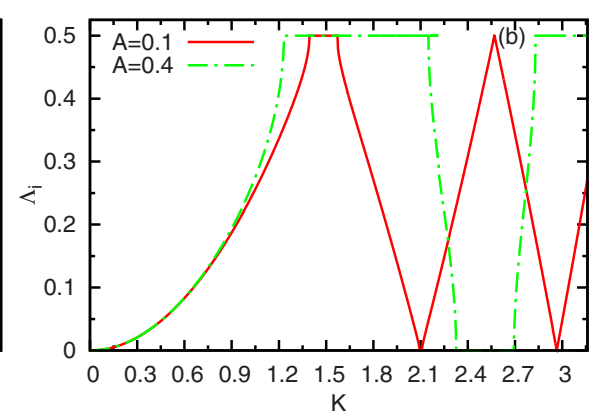

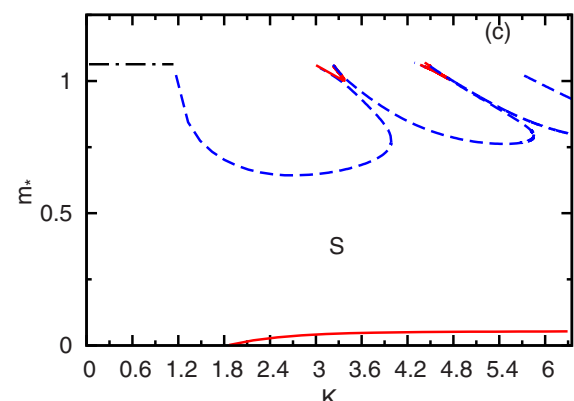

FIG. 2. (Color online) Numerical results at $\chi=-0.05$. Panel (a): neutral stability curves $m_{*}(K)$ for different values of the vibration amplitude $A$. Panel (b): imaginary part of the Floquet exponent, $\Lambda_{i}(K)$. Solid and dashed-dotted lines correspond to $A=0.1$ and $A=0.4$, respectively. Panel (c): marginal stability curves at $A=0.6$. Solid, dashed, and dashed-dotted lines correspond to synchronous, subharmonic, and quasiperiodic modes, respectively; the domain of stability is marked with $S$

$$
\rho=e^{K^{2} \Psi(\tau)} .
$$

Substituting this ansatz into Eq. (27), we obtain the equation in terms of the "eikonal" $\Psi(\tau)$

$$
\begin{aligned}
& \dot{\Psi}^{2}-\dot{\Psi} \xi_{1}-\xi_{2}+\frac{1}{K^{2}}\left(\ddot{\Psi}-\varphi_{\chi} A \sin \tau\right)=0, \\
& \xi_{1}=\alpha_{k}-\varphi_{\chi} A \cos \tau, \quad \xi_{2}=\Theta_{k}+\Phi_{k} A \cos \tau
\end{aligned}
$$

Dealing with the leading order of the expansion, we disregard the last term and arrive at

$$
\dot{\Psi}^{2}-\dot{\Psi} \xi_{1}-\xi_{2}=0
$$

The critical Marangoni number $m_{*}$ is determined by the condition

$$
\overline{\dot{\Psi}}=\frac{1}{2 \pi} \int_{0}^{2 \pi}\left[\alpha_{k}+\sqrt{\xi_{1}^{2}+4 \xi_{2}}\right] \mathrm{d} \tau=0 .
$$

Here the bar means the averaging over the period of the vibration.

Depending on the parameters two different situations are possible. If the integrand is real, this condition determines a stability threshold for the synchronous mode. In the more complicated case of the complex-valued integrand $\dot{\Psi}=\dot{\Psi}_{r}$ $+i \dot{\Psi}_{i}$, the quasiperiodic mode takes place. Over the vibration period, the perturbation fields are multiplied by $e^{i \phi}$, where $\phi$ is determined by

$$
\phi=K^{2} \int_{0}^{2 \pi} \Psi_{0}^{(i)} d \tau
$$

i.e., the gain in the phase $|\phi|$ is large.

In the further discussions these modes will be referred to as "short-wave synchronous" and "short-wave quasiperiodic" modes, respectively. The switching between these two modes occurs when the minimal value of the radicand in Eq. (73) is zero. The minimum can materialize at $\tau=\tau_{*}$, which is determined by the condition

$$
A \cos \tau_{*}=\frac{\alpha_{k} \varphi_{\chi}-2 \Phi_{k}}{\varphi_{\chi}^{2}},
$$

or at $\tau=0, \pi$, when $\cos \tau$ has an extremum. The former situation takes place at

$$
\Theta_{k}+\alpha \frac{\Phi_{k}}{\varphi_{\chi}}-\frac{\Phi_{k}^{2}}{\varphi_{\chi}^{2}}=0,
$$

whereas the latter one is at

$$
\left(\alpha_{k} \mp A \varphi_{\chi}\right)^{2}+4\left(\Theta_{k} \pm A \Phi_{k}\right)=0 .
$$

Our calculations show that when the short-wave quasiperiodic mode emerges for negative $\chi$ the condition given by $\mathrm{Eq}$. (76) is met; for positive $\chi$, the minimum takes place at $\tau$ $=0$ and correspondingly the condition given by Eq. (77) (with the upper signs) is valid.

For small $A$, the integral in Eq. (73) can be easily calculated

$$
m_{*} \approx \frac{L}{\chi_{L}}+A^{2} \frac{\Phi_{k}\left(\Phi_{k}-\varphi_{\chi} \alpha_{0}\right)}{2 \chi_{L} \alpha_{0}^{2}} .
$$

This result coincides with the short-wave limit of expression $m_{m}+m_{2 *} A^{2}$ found in Sec. IV A for small $A$, see Eq. (56) for $m_{2 *}$. Thus, the limits $A \rightarrow 0$ and $K \rightarrow \infty$ commute.

For finite $A$ numerical calculations of $m_{*}$ are performed.

\section{NUMERICAL RESULTS}

\section{A. Negative $\chi$}

In most of the calculations we keep fixed two of the parameters: $\varphi=1, \mathrm{~L}=0.01$, varying $\chi, m$, and $A$.

We start the discussion of the numerical results with negative $\chi$, when an oscillatory instability takes place in the absence of the vibration. Typical examples of the neutral stability curves are shown in Figs. 1(c) and 2. The first of these figures presents the comparison of numerical results with the analytical formula for small $A$. A good agreement is found for $A=0.05$.

In Fig. 2(a), neutral stability curves $m_{*}(K)$ are plotted for $\chi=-0.05$ and different values of the vibration amplitude. The horizontal parts of the lines correspond to the stability boundary for the quasiperiodic mode, the first and the third 

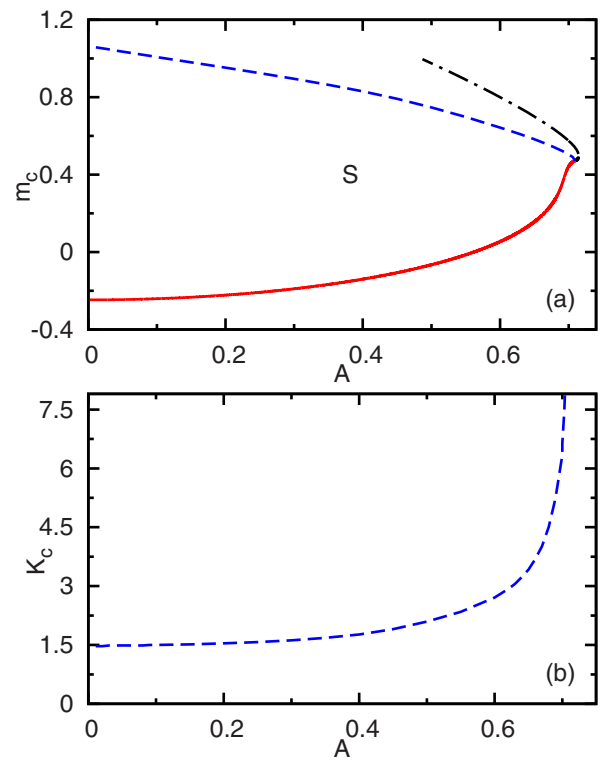

FIG. 3. (Color online) Stability domains $m_{c}(A)$ (a) and critical wavenumber $K_{c}(A)$ (b) for $\chi=-0.05$. Panel (a): solid, dashed, and dashed-dotted lines correspond to short-wave synchronous, subharmonic, and short-wave quasiperiodic modes respectively, the domain of stability is marked with $S$. Panel (b): $K_{c}$ for the subharmonic instability mode.

instability zones correspond to the subharmonic mode, the second one corresponds to the synchronous mode. It is clear from Fig. 2 that with increase in the amplitude the instability zones become larger. The critical wavenumber corresponding to the minimal Marangoni number, increases similarly to the case of small $A$, cf. Eq. (44). This shift is especially pronounced for the synchronous mode.

Panel (b) in Fig. 2 presents the variation of the imaginary part $\Lambda_{i}$ of the Floquet exponent along the neutral stability curves presented in panel (a). This plot confirms that the horizontal parts in Fig. 2(a) correspond to the quasiperiodic mode, whereas the resonant perturbations are subharmonic or synchronous, as $\Lambda_{i}=1 / 2$ or $\Lambda_{i}=0$ there.

Further increase in the amplitude leads to the emergence of a novel instability mode as clearly seen in Fig. 2(c). This mode is synchronous and it stems from the monotonic instability at negative $m$ for $A=0$. The critical wavenumber [corresponding to the maximum $\left.m_{*}(K)\right]$ is infinite. This instability mode exists even at zero Marangoni number, thus it is clear that it has a vibrational origin. It will be addressed in details in Sec. V C.

Now let us discuss the domains of stability on the plane $\left(A, m_{*}\right)$ for a fixed small negative value of the Soret number, see Fig. 3(a). With the growth in the vibration amplitude, the stability interval in $m_{*}$ becomes narrower and disappears at $A_{*} \approx 0.709$. At this point, the condition given by Eq. (76) is satisfied, i.e., the stability boundary for the short-wave synchronous mode intersects with that for the short-wave quasiperiodic one, as shown in Fig. 3(a). Exactly at $A=A_{*}$ the subharmonic mode branches from the quasiperiodic mode, since the condition $\phi=\pi$ is met in $K^{-2}$ vicinity of $A_{*}$. The critical wavenumber for this mode is infinite, when the branching occurs, but with decrease in $\chi$, it rapidly becomes of order of unity, see Fig. 3(b).
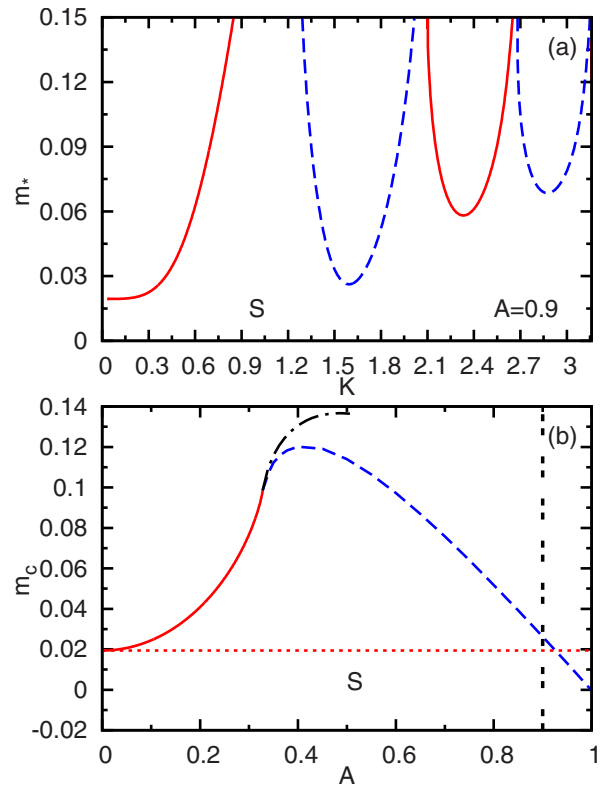

FIG. 4. (Color online) Panel (a): Neutral stability curves $m_{*}(K)$ at $A=0.9$, $\chi=0.5$. Solid lines correspond to the synchronous mode and dashed lines correspond to the subharmonic one. Panel (b): stability domain $m_{c}(A)$ at $\chi$ $=0.5$. Solid, dashed, and dashed-dotted lines correspond to the short-wave synchronous, subharmonic, and short-wave quasiperiodic modes, respectively. The domain of stability is marked with $S$. The vertical line in panel (b) shows $A=0.9$, see panel (a).

\section{B. Positive $\chi$}

For positive values of $\chi$ the situation is qualitatively different. In Fig. 4(a) typical neutral stability curves are demonstrated, and in Fig. 4(b), the stability domain is plotted. It is clear from Fig. 4, that the long-wave synchronous mode (with $K=0$ ) is critical at $A<0.925$. As it has been mentioned in Sec. IV B, $m_{c}=m_{m}$ in this case for any amplitude. At $A$ $=0.925$, the subharmonic mode becomes critical. This mode emerges at $A=0.328$ and $m_{c}=0.0985$, when the condition of intersection of two short-wave modes, Eq. (77), is satisfied. With the further growth in the vibration amplitude the critical Marangoni number decreases and becomes negative. Therefore, again the vibration-induced subharmonic mode takes place (cf. Fig. 5).

\section{Vibration-induced mode}

Now we discuss in more detail the results obtained for $M=0$, when the vibration is the only reason for the instability. This instability mode is usual in the thermal vibrational convection under weightlessness conditions. ${ }^{4}$

The results of calculations for this mode are shown in Fig. 5, where the stability domain in the plane $A-\chi$ is plotted. For negative $\chi$ the synchronous short-wave mode is critical, the critical value of the amplitude $A_{c}$ decreases with an increase in $|\chi|$. At $\chi=\chi_{*}$, which is very close to zero, again, an intersection of the stability boundaries for two short-wave modes, synchronous and quasiperiodic, occurs, and the subharmonic mode with large $K_{c}$ emerges. With the increase in $\chi$ the critical wavenumber for the subharmonic mode diminishes, whereas the value of $A_{c}$ slowly decreases. 

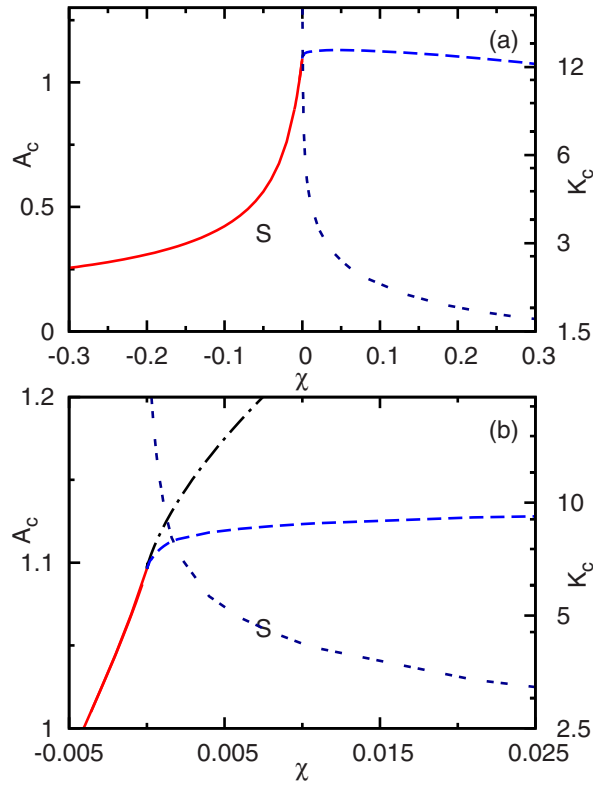

FIG. 5. (Color online) Critical vibration amplitude $A_{c}(\chi)$ at $m=0$. Solid and dashed lines correspond to short-wave synchronous and subharmonic modes, respectively, the domains of stability are marked with $S$. Doubledashed line corresponds to the critical wavenumber $K_{c}(A)$ (right logarithmic axis) for the subharmonic instability mode. Panel (b): fragment of panel (a) with the zoomed in area of the interaction of the two short-wave modes. Dashed-dotted line demonstrates $A_{c}(\chi)$ for the short-wave quasiperiodic mode.

\section{Diagrams for $m_{c}(\chi)$}

Summary of the results is presented in Fig. 6, where the stability domains $m_{c}(\chi)$ are shown for two fixed values of the vibration amplitude. In Fig. 6(a), critical stability curves without vibration ${ }^{30}$ are plotted for a comparison. As it can be seen, with the growth of the vibration amplitude the situation changes drastically.

For $A=0.5$, the stability domain is simply connected. For the negative Soret numbers, it is bounded from above by the stability curve corresponding to the subharmonic mode and from below by the stability curve for the synchronous shortwave mode, see Fig. 6(a). With an increase in $\chi$, the critical wavenumber grows for the subharmonic mode tending to the infinity at $\chi \approx 0$, when the dashed line ends up at the point of intersections of the lines corresponding to the quasiperiodic and synchronous short-wave modes, see Fig. 6(b). Within the interval of $\chi$ the short-wave synchronous mode becomes critical. At $\chi \approx 0.0104$ again the subharmonic mode emerges at the point of intersection of the lines corresponding to the short-wave quasiperiodic and synchronous modes. With further increase in $\chi$, the critical wavenumber for the synchronous mode diminishes fast from infinity, similar to that shown in Fig. 5(b).

For positive $\chi$ the stability domain is bounded only from above by the line corresponding to the long-wave synchronous mode. Obviously the margin for the long-wave synchronous perturbations (dotted line) coincides with margin for the long-wave monotonic perturbations for $A=0$ (pale solid line), since the former is not influenced by the vibration. For rather large values of $\chi, \chi>1.83$, which are beyond Fig. 6(a), the subharmonic mode becomes critical. This competition of the synchronous long-wave mode and the subharmonic mode is similar to that depicted in Fig. 4.

For larger vibration amplitude $A=0.8$, the stability region consists of two separate domains, see Fig. 6(c). One of them is situated at negative values of $\chi$; it is bounded by the stability curves corresponding to the subharmonic and shortwave synchronous modes. Another domain is mainly situated at positive values of $\chi$; it is bounded by the stability curves for the long-wave and short-wave synchronous perturbations. Starting from $\chi=0.829$ the subharmonic mode becomes critical instead of the long-wave synchronous one. With the further increase in $A$, the stability domains become smaller.

\section{CONCLUSIONS}

The impact of the vibration on the long-wave Marangoni convection is studied within the linear stability problem. Amplitude equation for density perturbation resembling the well-known Mathieu equation is derived. This equation is solved numerically, by means of the Floquet theory (Sec. V) and analytically for the limits of small vibration amplitude $A$ (Sec. IV A), large (Sec. IV C) and small (Sec. IV B) rescaled wavenumber $K$. In the latter case we show that the critical Marangoni number for $K=0$ does not depend on the amplitude of the vibration.

The instability modes are classified according to the value of the Floquet exponent at the stability boundary and
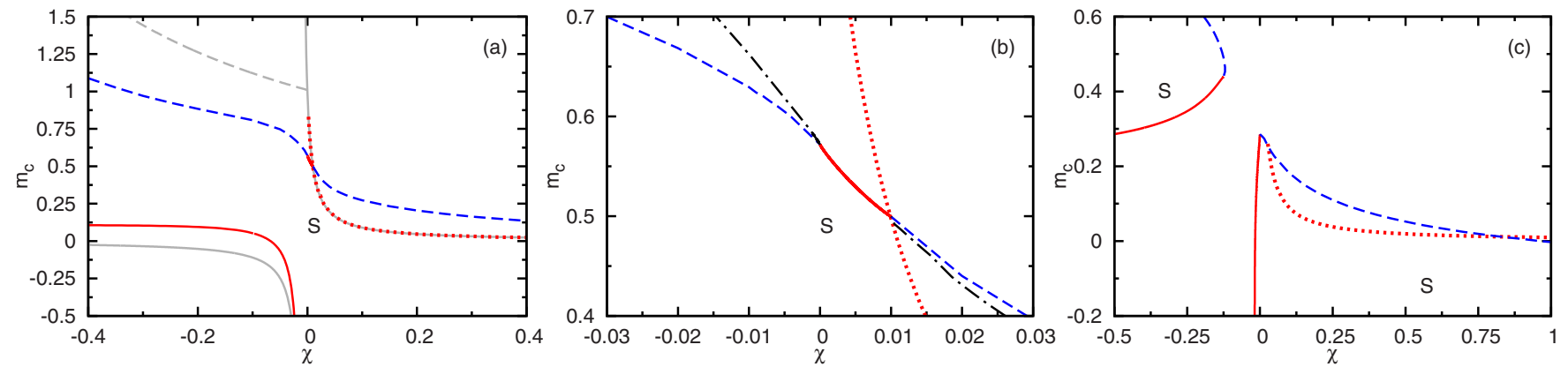

FIG. 6. (Color online) Stability domains $m_{c}(\chi)$. Dashed lines correspond to the subharmonic modes; dotted line is the stability margin for the long-wave synchronous perturbations, solid lines are the stability boundary for the short-wave synchronous mode, the domains of stability are marked with $S$. Panel (a): at $A=0.5$. Pale solid (dashed) lines correspond to neutral stability curves for monotonic (oscillatory) mode at $A=0$. Panel (b): fragment of panel (a) for small $|\chi|$. Dashed-dotted line corresponds to short-wave quasiperiodic mode here. Panel (c): at $A=0.8$. 
the critical value of $K_{c}$. The critical perturbations are (i) subharmonic ones with the finite critical wavenumber $K_{c}$, (ii) long-wave synchronous with $K_{c}=0$, and (iii) short-wave synchronous with $K_{c} \gg 1$.

A vibration-induced synchronous short-wave mode is also found at negative values of the Soret number $\chi$, see Fig. 5 . This mode originates from the monotonic mode for heating from above in the absence of vibration. At positive $\chi$, the subharmonic vibration-induced mode takes place.

The obtained results show that vibration destabilizes the layer of binary mixture with the Soret effect.

\section{ACKNOWLEDGMENTS}

The authors acknowledge the support of the Israel Science Foundation (Grant No. 812/06), and joint grants from the Israel Ministry of Sciences (Grant No. 3-5799) and the Russian Foundation for Basic Research (Grant No.09-0192472). The work is also supported by the European Union via FP7 Marie Curie scheme Grant No. PITN-GA-2008214919 (MULTIFLOW).

\section{APPENDIX A: CONDITION OF NONDEFORMABLE FREE SURFACE}

Here, we briefly discuss the basic assumptions underlying Eqs. (4)-(9). The first group of the limitations is the conventional restrictions on the gravity and gravity-induced buoyancy

$$
\mathrm{Ga}_{P} \gg \mathrm{M} \gg \mathrm{Ga}_{P} \max \left(\beta_{T}\left|a_{*}\right| d_{*},\left|\beta_{C} a_{*} \alpha_{*}\right| d_{*}\right),
$$

where $\mathrm{Ga}_{P} \equiv g_{*} d_{*}^{3} /\left(\nu_{*} \kappa_{*}\right)=\mathrm{GaP}$ and Ga is the Galileo number ( $g_{*}$ is the gravity acceleration). The former inequality allows us to neglect the surface deflection, whereas the latter one is sufficient to disregard the buoyancy convection. Below we denote $\max \left(\beta_{T}\left|a_{*}\right| d_{*},\left|\beta_{C} a_{*} \alpha_{*}\right| d_{*}\right)$ as the Boussinesq parameter $\delta$.

Also we assume $b_{*} \Omega_{*}^{2} \gg g_{*}$ or equivalently,

$$
\mathrm{Ga}_{P v}=\mathrm{PGa}_{v} \equiv \frac{b_{*} \Omega_{*}^{2} d_{*}^{3}}{\nu_{*} \kappa_{*}} \gg \mathrm{Ga}_{P},
$$

in such a way that $b=\mathrm{Ga}_{P v} \delta$ becomes finite.

Keeping in mind that $\omega=O\left(\varepsilon^{2}\right)$ and $\mathrm{P}^{2} \mathrm{Ga}_{v}=B \omega^{2} \gg 1$, we arrive at the following estimation for the dimensionless amplitude of vibration $B$ :

$$
B \equiv \frac{b_{*}}{d_{*}}=O\left(\varepsilon^{-4} / \delta\right) .
$$

Applying the large-amplitude vibration we have to ensure that no Faraday instability occurs in the system. To prevent the instability, we assume that the surface tension is large enough $\mathrm{Ca}=\sigma^{(0)} d_{*} /\left(\nu_{*}^{2} \rho^{(0)}\right) \gg \mathrm{Ga}$, which is valid for cases B1.2 (long-wave limit) or B1.3 (small-frequency limit) in Ref. 9. Our limitations mean that $\widetilde{\omega}=\mathrm{P}^{-1} \omega \ll 1$ and $\widetilde{a}$ $=B / \sqrt{\mathrm{Ca}}$, where the dimensionless frequency $\widetilde{\omega}=\Omega_{*} d_{*}^{2} \nu_{*}^{-1}$ and amplitude $\tilde{a}=b_{*} \nu_{*} / d_{*} \sqrt{\sigma^{(0)} d_{*} / \rho^{(0)}+g_{*} d_{*}^{3}}$ of vibration were defined in the cited paper. Application of the stability conditions from Ref. 9 yields the critical dimensionless acceleration:

$$
\frac{B_{c} \omega^{2}}{\sqrt{\mathrm{Ca}}}=\widetilde{a}_{c} \widetilde{\omega}^{2}=A_{c}^{(M V)}(\gamma), \quad \gamma=\frac{\mathrm{Ga}}{\sqrt{\mathrm{Ca}}} .
$$

The function $A_{c}^{(M V)}(\gamma) /(1+\gamma)$ is shown in Fig. 5(d) of the cited paper; it decays monotonically from 8.5 to 1.5 as $\gamma$ grows from zero to infinity. Therefore, at $\delta \sqrt{\mathrm{Ca}}=O(1)$ there is no Faraday instability under the condition

$$
b<A_{c}^{(M V)} \mathrm{P} \sqrt{\mathrm{Ca}} \delta .
$$

Consider a layer of water mixture of ethanol of thickness $d_{*}=0.03 \mathrm{~cm}$ under the temperature difference $1 \mathrm{~K}$. The typical values of the dimensionless parameters are as follows: $\mathrm{M} \approx 10^{2}, \mathrm{Ga}_{P} \approx 10^{3}, \mathrm{Ca} \approx 10^{4}, \mathrm{P} \approx 10$, and $\delta \approx 10^{-3}$. Therefore, the estimations provided by Eq. (A1) are met and the value $b \approx 10$ are available according to Eq. (A5). The values of the dimensional amplitude and frequency for this setup can be chosen, for instance, $b_{*} \approx 30 \mathrm{~cm}$ and $\Omega_{*}$ $\approx 0.1 \mathrm{rad} / \mathrm{s}$, respectively, which seems to be easily materialized in experiments.

\section{APPENDIX B: SET OF EQUATIONS AND THE SOLVABILITY CONDITION}

The set of equations and boundary conditions in the second order of approximation is written as

$$
\frac{\partial^{4} \widetilde{\Psi}_{2}}{\partial z^{4}}=\frac{1}{\mathrm{P}} \frac{\partial}{\partial \tau} \frac{\partial^{2} \widetilde{\Psi}_{0}}{\partial z^{2}}+2 K^{2} \frac{\partial^{2} \widetilde{\Psi}_{0}}{\partial z^{2}}+i K\left(\widetilde{T}_{2}+\varphi \widetilde{C}_{2}\right) b \cos \Omega \tau,
$$

$$
\begin{aligned}
& \frac{\partial^{2} \widetilde{T}_{2}}{\partial z^{2}}=\frac{\partial \widetilde{T}_{0}}{\partial \tau}+i K \widetilde{\Psi}_{0}+K^{2} \widetilde{T}_{0}, \\
& \frac{\partial^{2}}{\partial z^{2}}\left(\widetilde{C}_{2}+\chi \widetilde{T}_{2}\right)=\frac{1}{\mathrm{~L}}\left(\frac{\partial \widetilde{C}_{0}}{\partial \tau}-i K \chi \widetilde{\Psi}_{0}\right)+K^{2}\left(\widetilde{C}_{0}+\chi \widetilde{T}_{0}\right),
\end{aligned}
$$

$$
\begin{aligned}
& \widetilde{\Psi}_{2}=\frac{\partial \widetilde{\Psi}_{2}}{\partial z}=\frac{\partial \widetilde{T}_{2}}{\partial z}=\frac{\partial \widetilde{C}_{2}}{\partial z}=0 \text { at } z=0, \\
& \widetilde{\Psi}_{2}=\frac{\partial \widetilde{T}_{2}}{\partial z}=\frac{\partial \widetilde{C}_{2}}{\partial z}=0, \\
& \frac{\partial^{2} \widetilde{\Psi}_{2}}{\partial z^{2}}=-i K \mathrm{M}\left(\widetilde{T}_{2}-\widetilde{C}_{2}\right) \text { at } z=1 .
\end{aligned}
$$

The solvability condition in the second order yields

$$
\frac{\partial}{\partial \tau}\left(\begin{array}{c}
\left\langle\widetilde{T}_{0}\right\rangle \\
\mathrm{L}^{-1}\left\langle\widetilde{C}_{0}\right\rangle
\end{array}\right)=i K\left(\begin{array}{c}
-1 \\
\chi \mathrm{L}^{-1}
\end{array}\right)\left\langle\widetilde{\Psi}_{0}\right\rangle-K^{2}\left(\begin{array}{c}
\left\langle\widetilde{T}_{0}\right\rangle \\
\left\langle\widetilde{C}_{0}\right\rangle+\chi\left\langle\widetilde{T}_{0}\right\rangle
\end{array}\right),
$$

where $\langle\cdots\rangle=\int_{0}^{1} \cdots d z$ means an averaging over the layer thickness.

${ }^{1}$ T. Frommelt, M. Kostur, M. Wenzel-Schäfer, P. Talkner, P. Hänggi, and A. Wixforth, "Microfluidic mixing via acoustically driven chaotic advection," 
Phys. Rev. Lett. 100, 034502 (2008).

${ }^{2}$ G. Barenblatt, V. Entov, and V. Ryzhik, Theory of Fluid Flows Through Natural Rocks (Kluwer, Dordrecht, 1990).

${ }^{3}$ N. Riley, "Steady streaming," Annu. Rev. Fluid Mech. 33, 43 (2001).

${ }^{4}$ G. Z. Gershuni and D. V. Lyubimov, Thermal Vibrational Convection (Wiley, New York, 1998).

${ }^{5}$ D. V. Lyubimov, T. P. Lyubimova, and A. A. Cherepanov, Dynamics of Interfaces in Vibration Fields (Fizmatlit, Moscow, 2004) (in Russian).

${ }^{6}$ V. Kozlov, A. Ivanova, and P. Evesque, "Sand behaviour in cavity with incompressible liquid under vertical vibrations," Europhys. Lett. 42, 413 (1998).

${ }^{7}$ U. Thiele, J. M. Vega, and E. Knobloch, "Long-wave Marangoni instability with vibrations," J. Fluid Mech. 546, 61 (2006).

${ }^{8} \mathrm{M}$. Faraday, "On a peculiar class of acoustic figures; and on certain forms assumed by groups of particles upon vibrating elastic surfaces," Philos. Trans. R. Soc. London 121, 299 (1831).

${ }^{9}$ F. J. Mancebo and J. M. Vega, "Faraday instability threshold in largeaspect-ratio containers," J. Fluid Mech. 467, 307 (2002).

${ }^{10} \mathrm{G}$. Venezian, "Effect of modulation on the onset of thermal convection," J. Fluid Mech. 35, 243 (1969).

${ }^{11}$ J. R. Rogers, W. Pesch, O. Brausch, and M. F. Schatz, "Complex-ordered patterns in shaken convection," Phys. Rev. E 71, 066214 (2005).

${ }^{12}$ Y. Shu, B. Q. Li, and B. R. Ramaprian, "Convection in modulated thermal gradients and gravity: Experimental measurements and numerical simulations," Int. J. Heat Mass Transfer 48, 145 (2005).

${ }^{13}$ P. M. Gresho and R. L. Sani, "The effect of gravity modulation on the stability of a heated fluid layer," J. Fluid Mech. 40, 783 (1970).

${ }^{14}$ G. Z. Gershuni, E. M. Zhukhovitskii, and I. S. Iurkov, "On the convective stability in the presence of periodically varying parameter," J. Appl. Math. Mech. 34, 442 (1970).

${ }^{15}$ B. I. Myznikova and B. L. Smorodin, "Convective instability of a horizontal binary-mixture layer in a modulated external force field," Fluid Dyn. 36, 1 (2001).

${ }^{16}$ B. I. Myznikova and B. L. Smorodin, "Longwave instability of a binary mixture flow in a vertical channel in the presence of vibration," Fluid Dyn. 44, 240 (2009).
${ }^{17}$ B. L. Smorodin, B. I. Myznikova, and I. O. Keller, "On the Soret-driven thermosolutal convection in a vibrational field of arbitrary frequency," Lect. Notes Phys. 584, 372 (2002).

${ }^{18}$ B. L. Smorodin, "Convection of a binary mixture under conditions of thermal diffusion and variable temperature gradient," J. Appl. Math. and Tech. Phys. 43, 217 (2002).

${ }^{19}$ B. L. Smorodin, G. Z. Gershuni, and M. G. Velarde, "On the parametric excitation of thermoelectric instability in a liquid layer open to air," Int. J. Heat Mass Transfer 42, 3159 (1999).

${ }^{20}$ R. V. Birikh, V. A. Briskman, A. A. Cherepanov, and M. G. Velarde, "Faraday ripples, parametric resonance, and the Marangoni effect," J. Colloid Interface Sci. 238, 16 (2001).

${ }^{21}$ J. R. L. Skarda, "Instability of a gravity-modulated fluid layer with surface tension variation," J. Fluid Mech. 434, 243 (2001).

${ }^{22}$ A. C. Or and R. E. Kelly, "The effect of thermal modulation upon the onset of Marangoni-Bénard convection," J. Fluid Mech. 456, 161 (2002).

${ }^{23}$ B. L. Smorodin, A. B. Mikishev, A. A. Nepomnyashchy, and B. I. Myznikova, "Thermocapillary instability of a liquid layer under heat flux modulation," Phys. Fluids 21, 062102 (2009).

${ }^{24}$ V. I. Yudovich, S. M. Zenkovskaya, V. A. Novossiadliy, and A. L. Shleykel, "Parametric excitation of waves on a free boundary of a horizontal fluid layer," C. R. Mec. 332, 257 (2004).

${ }^{25}$ S. M. Zen'kovskaya, V. A. Novosyadlyi and A. L. Shleikel, "The effect of vertical vibration on the onset of thermocapillary convection in a horizontal liquid layer," J. Appl. Math. Mech. 71, 247 (2007).

${ }^{26}$ P. Colinet, J.-C. Legros, and M. G. Velarde, Nonlinear Dynamics of Surface-Tension-Driven Instabilities (Wiley-VCH, Berlin, 2001).

${ }^{27}$ A. A. Nepomnyashchy, M. G. Velarde, and P. Colinet, Interfacial Phenomena and Convection (Chapman and Hall/CRC, London/Boca Raton, FL, 2001).

${ }^{28}$ R. V. Birikh, V. A. Briskman, M. G. Velarde, and J.-C. Legros, Liquid Interfacial Systems. Oscillations and Instability (Dekker, New York, Basel, 2003).

${ }^{29}$ A. H. Nayfeh, Perturbation Methods (Wiley, New York, 1973).

${ }^{30}$ A. Oron and A. A. Nepomnyashchy, "Long-wavelength thermocapillary instability with the Soret effect," Phys. Rev. E 69, 016313 (2004). 